\title{
Ad Hoc Link Traversal Time Prediction
}

\author{
Rutger Claes and Tom Holvoet
}

\begin{abstract}
Drivers able to use link traversal time predictions can use this information to choose better routes. Such anticipatory vehicle routing bases routing decisions on link traversal times likely to be experienced by the vehicle instead of historic or real time link traversal times. However, generating such link traversal times is a challenge. This paper describes a multiagent system in which road agents and vehicle agents cooperate to provide ad hoc link traversal times for the routes the vehicle agents intend to follow. Road agents use observations to learn a model of the road they represent and combine this model with information obtained from vehicle agents to predict future link traversal times. Two such models are described in this paper, namely a polynomial model and an artificial neural network. Both models are evaluated based on a microscopic traffic simulation. The predictions obtained using both models are analyzed and compared with the actual observed link traversal times to estimate the accuracy of the predictions.
\end{abstract}

\section{INTRODUCTION}

Vehicle drivers often get route decision support from their satellite navigation systems. Usually the satellite navigation system calculates one or more routes to the driver's destination and then proceeds to guide the driver along that route. While calculating these routes, the satellite navigation system takes into account the static characteristics of the traffic network to come up with the times needed to travel along each of the links in the route. The latest generation of satellite navigation systems are equipped with communication capabilities and go even further. By receiving real-time information about the current traffic situation the satellite navigation system software can improve on its original estimates based on static information. The route calculation process thus becomes based on more accurate link traversal times and yields better results [1].

Using real-time traffic information in the route calculation process assumes that the link traversal times will remain stable. If the link traversal times change between the moment the route is calculated and the moment the vehicle actually traverses the link, the resulting route could be suboptimal. Dynamic rerouting can reduce this problem. However, frequent or myopic route changing also negatively impacts the quality of the route. A possible solution to this problem is the use of predicted link traversal times. Assuming the prediction

This research was funded by the IWT - SBO project 'MASE' (project no. 060823) and by the Interuniversity Attraction Poles Programme Belgian State, Belgian Science Policy, and by the Research Fund K.U.Leuven.

R. Claes is with DistriNet Labs, Department of Computer Science, Katholieke Universiteit Leuven, Belgium rutger.claes@cs.kuleuven. be

T. Holvoet is with DistriNet Labs, Department of Computer Science, Katholieke Universiteit Leuven, Belgium tom.holvoetacs.kuleuven.be of a link traversal time will be more stable than the real-time information, route quality can be improved [2].

A second benefit of using predicted link traversal times and anticipatory vehicle routing is the resulting distribution of traffic. In their paper [1], Wunderlich et al. show that when a fraction of all drivers participate in an anticipatory vehicle routing system, all drivers in the network benefit. By avoiding congested links, the participating drivers improve their own route quality and, at the same time, reduce the congestion for non-participating drivers. Anticipatory vehicle routing thus helps avoiding congestion.

Forecasting traffic situations in general is actively researched [1], [3], [4]. The approach taken in this paper differs from most existing research in that it bases predictions on information obtained from drivers participating in the system and observations made by the system itself. The system consists of cooperating autonomous software entities - or software agents - that are distributed across the traffic network. Together, these software agents form a multi-agent system. There are two types of agents in this multi-agent system responsible for predicting link traversal times, namely vehicle agents and road agents.

Road agents each represent a single link in the network infrastructure. These agents maintain a model describing the number of vehicles entering that link and the time it will take those vehicles to traverse the link. This model is fine-tuned using machine learning techniques. By monitoring the road they are responsible for, road agents train the model using observations of actual link traversal times.

Vehicle agents can be considered similar to the satellite navigation software. They travel along with the vehicles they represent. Vehicle agents have knowledge about the route the vehicle driver intends to follow. They communicate this information with all road agents representing links on that route. Assuming the vehicle agents know when they reach a particular link, that estimated time of arrival allows them to notify the road agent of their pending visit. The road agent in turn can aggregate these notifications and use these as input to the model it maintains, thus providing the vehicle agents with ad hoc generated link travel time predictions when requested. Each prediction obtained from a road agent is then used to calculate the estimated arrival time at the next link on the vehicle agents route. The functionality of such a multi-agent system is described in [5].

This paper focusses on the road agents and in particular, the model they use to predict link traversal times based on incoming notifications. Two techniques to model this relationship are evaluated. A first technique models the relationship as a simple mathematical function and using 
machine learning techniques to learn the parameters of this mathematical function. A second technique models the relationship as a black box using artificial neural networks.

Both techniques are evaluated using microscopic traffic simulations. In these traffic simulations, the respective models are used to generate ad hoc predictions of link traversal times. Both the predictions and the actual link traversal times are recorded and allow a comparison between predicted and actual traversal times.

The remainder of this paper is structured as follows. First, detailed descriptions of both of the modeling techniques and their implementations are offered in Section II. Section III compares both of the techniques with other techniques described in literature. Then Section IV describes the experimental setup used to evaluate both techniques. Next the results of several experiments are presented in Section V. Based on the results of these experiments, some conclusions and future work is presented in Section VI.

\section{TRAFFIC PREDICTION MODELS}

The advanced traffic information system described in [5] relies on road agents that are capable of predicting link traversal times for all links in the network. Vehicle agents provide the road agents with information about the route they intent to follow. Road agents use this information to generate link traversal time predictions. Predictions are generated ad hoc based on the information available to the road agent whenever a vehicle agent sends a request.

In this paper, we explore two approaches to generate these ad hoc predictions based on information obtained from vehicle agents. A first approach evaluated in this paper models the road based on a "white box" approach. It assumes a polynomial relationship between the number of vehicles entering the road and their traversal time. The second approach uses artificial neural networks as a "black box" representation of the relationship between vehicles entering and their traversal time. The model in the first approach is a polynomial function and can be interpreted by traffic network experts. The models resulting from the second approach, however, have no meaning and completely rely on machine-learning techniques.

Both models are trained by road agents using the same information. Every road agent monitors the road for which it maintains the model and observes vehicles entering and exiting the link. Whenever a vehicle exits the link, the road agent calculates the time that vehicle actually needed to traverse the link and cross references the vehicles arrival time for the number of notifications received. Such an observation is then used as training data to train the models.

\section{A. Polynomial Model}

The polynomial model describes the relationship between the link traversal times and the number of notifications at the vehicles arrival time. The only parameter in this model is the degree of the polynomial.

Training the model consists of collecting training points and running a regression over them. The training points can be weighted based on how long ago they occurred. In the implementation used in this paper, no such weighting is used. A Gauss Newton optimizer from the Apache Commons Java framework [6] was used to perform the actual regression.

Evaluating the model to predict the link traversal time at some time in the future is as simple as evaluating the learned polynomial function for the number of notifications received for that time.

\section{B. Artificial Neural Network based models}

The approach taken by a road agent when modeling the relationship link traversal time and notifications differs somewhat from the approach taken with the polynomial models. Artificial neural networks make it easy to include additional inputs into the model. To account for traffic possibly building up on the road, the neural network based model also takes the number of notifications received at certain times before the vehicles arrival into account.

The most important parameters determining the neural network model include the network layout, the learning method and the transfer function. For this evaluation we have experimented with neural networks with one or two hidden layers. The layouts are $(8,17,1),(16,33,1)$ and $(8,17,17,1)$. A layout of $\left(l_{1}, l_{2}, \ldots, l_{n}\right)$ represents an artificial neural network with $n$ layers where the number of perceptrons in layer $k$ are $l_{k}$. The first integer determines the number of inputs and therefore also how many notification levels before the vehicles arrival time are taken into account. An input size of 8 , for example means the input of the neural network is the notification level at times eta $-i^{2}$ with $i$ in $0: 7$ and $e t a$ the estimated arrival time for the vehicle. The last number in the layout represents the number of outputs. The number of outputs of the neural network is always 1 , representing the duration. Such a neural network is illustrated in Figure 1.

At runtime, the neural network is trained using a back propagation algorithm. The implementation used for the neural network in the experiments described in this paper is the Neuroph library[7].

Unlike some other approaches described in Section III, the approach described in this paper does not rely on a sequential neural network starting from the current traffic situation.

\section{RELATED WORK}

\section{A. Collaborative traffic forecasting}

In [8], Lee et al. describe a collaborative traffic information generation and sharing system. The system described in [8] bares similarities with the ad hoc link travel time predictions described in this paper. In their paper, Lee et al. argue that traditional traffic information techniques such as sensor based, site based and probing vehicle based information collection is unable to collect sufficient information due to coverage and cost problems that remain unsolved. Therefor they propose a collaborative system where vehicles themselves contribute information. 


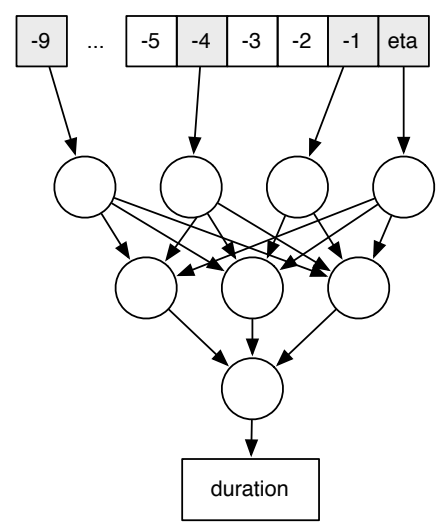

Fig. 1: Illustration of how a neural network with a $(4,3,1)$ layout would look like. The array at the top represents the number of notifications received at a vehicles estimated time of arrival and before. The output layer of the neural network gives the duration.

The system consists of three components, namely a Smart Traffic Agent (STA), the Real-Time Traffic Information Exchange Protocol (TIEP) and a centralized Traffic Information Center (TIC) that acts as a backend. The STA's resemble the vehicle agents used in this paper. STA's send out information about their immediate surroundings using the TIEP protocol. Information from vehicles and other traffic information sources is aggregated at the TIC. This TIC combines the realtime information with predictions generated by an expert rule based system. A combination of real-time information and forecasts is then sent back to the Smart Traffic Agents.

Although the concept of STA's resembles our use of vehicle agents and both approaches can be considered collaborative as they rely on information contributed by vehicles, the approach taken by Lee et al. differs from the approach described in this paper. There are three key differences between both approaches.

The first key difference is an architectural difference. The TIC aggregating the collaborative information and generating the predictions in the approach by Lee et al. is described as a centralized entity. In that sense, the approach described by Lee et al. resembles that of [1]. The approach used in this paper allows for a decentralized aggregation of information and prediction generation, which we believe is a benefit in a large scale environment such as traffic.

The second key difference is the fusion between real-time and forecast information. The system described by Lee et al. returns real-time traffic information about the immediate vicinity of the vehicle and forecast information about links further away. In our approach, such distinction is unnecessary as all information returned to the vehicle agent describes traffic conditions at the time the vehicle would experience it.

A third key difference is the information prediction process itself. In our approach we combine observations made by the road agent with vehicles intentions to generate link traversal time predictions. The information prediction process by Lee combines both internal information contributed by Smart
Traffic Agents and external data sources, such as agencies and other traffic information centers using a rule based expert system.

\section{B. Neural network based traffic forecasting}

In [4], Yasdi describes a method of short term road traffic prediction using a neural network based approach. The approach taken by Yasdi differs in a number of ways from the neural network based model used in this paper.

To take into account the temporal aspect of the traffic that is being predicted, Yasdi uses partially recurrent networks. By doing so, the neural network takes into account information it itself generated for previous times. The resulting neural networks state is dependent not only on the current input, but also on an aggregate of previous states. The neural network maintained by the road agents in this paper operate differently as they do not take into account previously generated outputs of the neural network to account for the temporal aspect, but instead use notification levels occurring before the vehicles' arrival.

A second difference between the approach described in [4] and that of this paper is the goal of the neural networks. In [4], the goal is to predict traffic levels while in the approach taken in this paper, future traffic levels are assumed to be known in the form of the notification levels. The goal of the neural networks used here is to obtain link traversal time predictions for individual vehicles.

\section{JamBayes}

In their paper [9], Horvitz et al. describe the predictive component "JamBayes" in their ATIS application "Smartphlow". Smartphlow is an application that can show the current traffic situation in the Greater Seattle area. The basic information shown in Smartphlow is based on traffic status reported by a network of sensors operated by the Washington Department of Transportation. Smartphlow can also predict problems in predefined commuter hotspots. For a set of 22 traffic bottleneck, Smartphlow can predict the expected time until a bottleneck experiencing a traffic jam will flow smoothly again, and the time until smoothly running bottlenecks become congested.

The approach taken by Horvitz et al. in JamBayes and Smartphlow differs from the approach taken in this paper. Besides the obvious difference in prediction model Bayesian networks are not evaluated in this paper - the focus of the work is different. JamBayes offers a static statistical model describing the possibilities that traffic congestion occurs at certain predefined hotspots. The JamBayes model is global in the sense that it encompasses and describes the entire network of bottlenecks in the Greater Seattle area. Our approach builds and learns a model for every link in the network. The models are completely isolated from each other because of the distributed nature of the environment. Furthermore, the predictions made by the JamBayes model are based on real time and real world data, whereas the predictions made in our approach are based on drivers intentions. 


\section{EXPERIMENT SETUP}

An experiment was created to evaluate the accuracy of both the polynomial and neural network based models. In this experiment, information from the Belgian city of Antwerp was extracted from OpenStreetMap [10] and loaded into a microscopic traffic simulation. The microscopic traffic simulation used is part of the GridLock traffic simulation platform [11]. The roads in the network were all assigned a road agent. Traffic consisting of vehicles equipped with vehicle agents are then simulated for a time period of 1 hour. During this hour, all observations and predictions made by the road agents are logged and stored for later analysis.

The simulation platform used is microscopic and combines the Intelligent Driver Model [12] and MOBIL lane changing model [13] to realistically simulate traffic. Because of the Intelligent Driver Model, effects such as stop and go waves caused by increasing levels of traffic are present in the simulation.

All simulations use the same origin destination matrix describing when and where a vehicle enters the network along with its destination. Upon entering the network a vehicle uses an $A^{*}$ based algorithm to calculate a route to its destination. This route calculation process does not use the link travel time predictions offered by the road agents. Vehicles in the simulation periodically (every 60 seconds) inform all road agents on their route of their trajectory. When such a notification arrives at a road agent, it generates a prediction. This prediction is logged for later offline analysis.

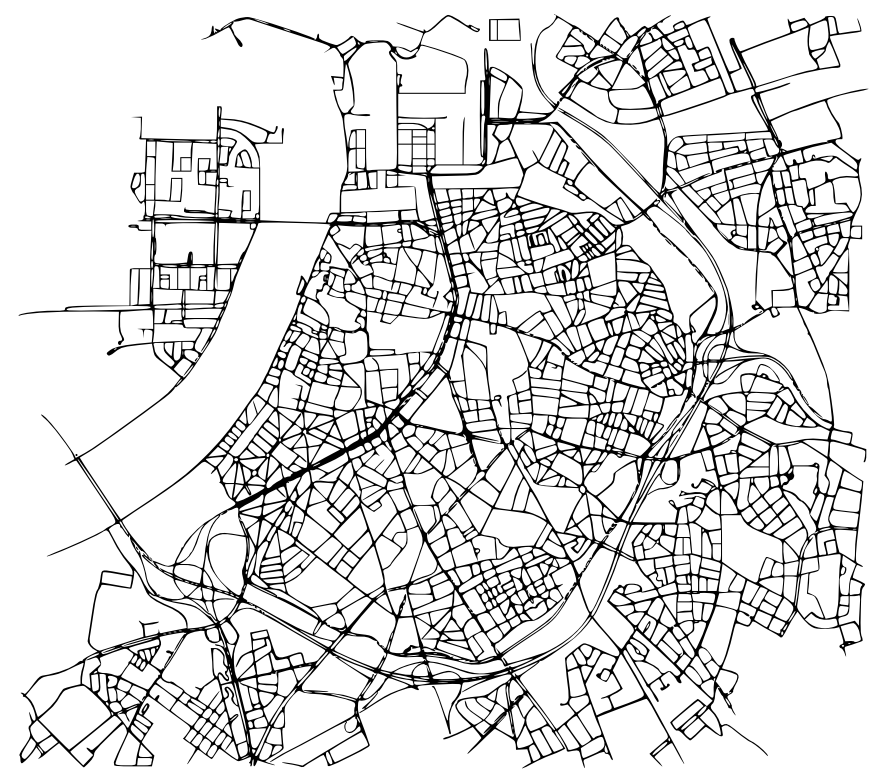

Fig. 2: Traffic network of the city of Antwerp as imported from OpenStreetMap data

Four different scenarios were simulated on the network shown in Figure 2. Each scenario is characterized by how the vehicles introduced in the network behave and the frequency of vehicle insertions.

The vehicles behavior is determined by the parameters in both the Intelligent Driver Model and the MOBIL lane changing model. By adjusting parameters such as the safe headway time or the minimal bumper to bumper distance a heterogenous driver behavior can be achieved. In the experiments two types of behaviors are used, namely uniform behavior where all vehicles have the same set of parameters and mixed behavior where vehicles are parameterized as normal vehicles driven by either a cautious or an aggressive driver or trucks. To simulate trucks, lower acceleration and longer vehicle lengths are combined with the cautious driver parameters. To evaluate the proposed approach using either the homogenous or heterogenous vehicle population, two copies of the origin destination matrix where made. In each of these copies, the vehicles descriptions is extended with the parameters used in the vehicles Intelligent Driver Model and MOBIL lane changing model. For the homogenous vehicle population all vehicles receive the same parameters, for the heterogenous population all vehicles receive different parameters based on the profile they belong to.

Table I shows the parameters used in both the MOBIL and IDM models for four driver profiles, namely neutral, cautious, reckless and truck drivers. The homogenous population consists solely of neutral drivers while the heterogenous population combines all four in equal proportions. Most of the model parameters are derived from two key parameters, namely the vehicle length $l$ and politeness $p$. This ensures variation between drivers while maintaining consistency between the parameters defining one driver. The two key parameters are chosen from a normal distribution of which mean and deviation can be found in Table I as $\mu \pm \sigma$. To ensure model validity, negative samples from the normal distribution are rejected and replaced by 0 .

TABLE I: Driver profiles based on MOBIL and IDM parameters

\begin{tabular}{|c||c|c|c|c|}
\hline Profile & Neutral & Cautious & Reckless & Truck \\
vehicle length $l$ & $3 \pm .5$ & $3 \pm .5$ & $3 \pm .5$ & $10 \pm 3$ \\
politeness $p$ & $0.2 \pm .1$ & $0.4 \pm .1$ & $0.1 \pm .05$ & $0.7 \pm .1$ \\
\hline acceleration $a$ & \multicolumn{3}{|c|}{$-0.08 \times l+2.16$} \\
deceleration $b$ & \multicolumn{3}{|c}{$-0.08 \times l+2.16$} \\
minimum gap $s_{0}$ & \multicolumn{3}{|c}{$0.1 \times l+1.8$} \\
time headway $T$ & \multicolumn{3}{|c}{$1.6 \times p+0.4$} \\
threshold $a_{\text {thr }}$ & \multicolumn{3}{|c}{$0.9 \times p+0.1$} \\
bias $\delta$ & \multicolumn{3}{c}{$0.4 \times p+0.1$} \\
\hline
\end{tabular}

Besides varying the drivers behavior a second variation point was defined. Both of the origin destination matrices describing the homogenous and heterogenous vehicle populations are again copied. In the copies, the rate at which the vehicles are introduced in the traffic network is altered to be more dynamic. Instead of a continuous inflow of traffic, it reshapes the existing inflow into a variable traffic inflow with relatively calm and busy periods. In the constant scenario's, vehicles are inserted as to maintain a constant number of 1000 vehicles in the network. In the variable scenario, the desired number of vehicles is modulated using a sine function with a period of 30 minutes and an amplitude of 333 vehicles. The number of vehicles in the network will thus fluctuate 
between 667 and 1333 vehicles.

Combined these two variation points, vehicle behavior and vehicle generation, result in four different scenarios in which we evaluate the descriptive road models, namely homogenous constant traffic, homogenous variable traffic, heterogenous constant traffic and heterogenous variable traffic. The experiment evaluates descriptive models using artificial neural networks with layouts $(8,17),(8,17,17)$ and $(16,33)$ using the principle illustrated in Figure 1 and polynomial models with a degree of 0,1 and 2 as shown in Figure ??.

\section{RESULTS}

In evaluating the performance of the different descriptive models we focus on the error of the predictions they generate. We define the normalized square error on a prediction as follows:

$$
e_{n}=\sqrt{\left(\frac{t_{o}-t_{p}}{t_{t}}\right)^{2}}
$$

. Where $t_{o}$ is the observed traversal time, $t_{p}$ is the predicted traversal time and $t_{t}$ is the theoretical traversal time based on static network information.

Figure 3 shows an overview of the mean square error on the predictions for all six models in all four scenarios. The figure clearly shows that the mean square error for the artificial neural network based models are lower than those of the polynomial based models.

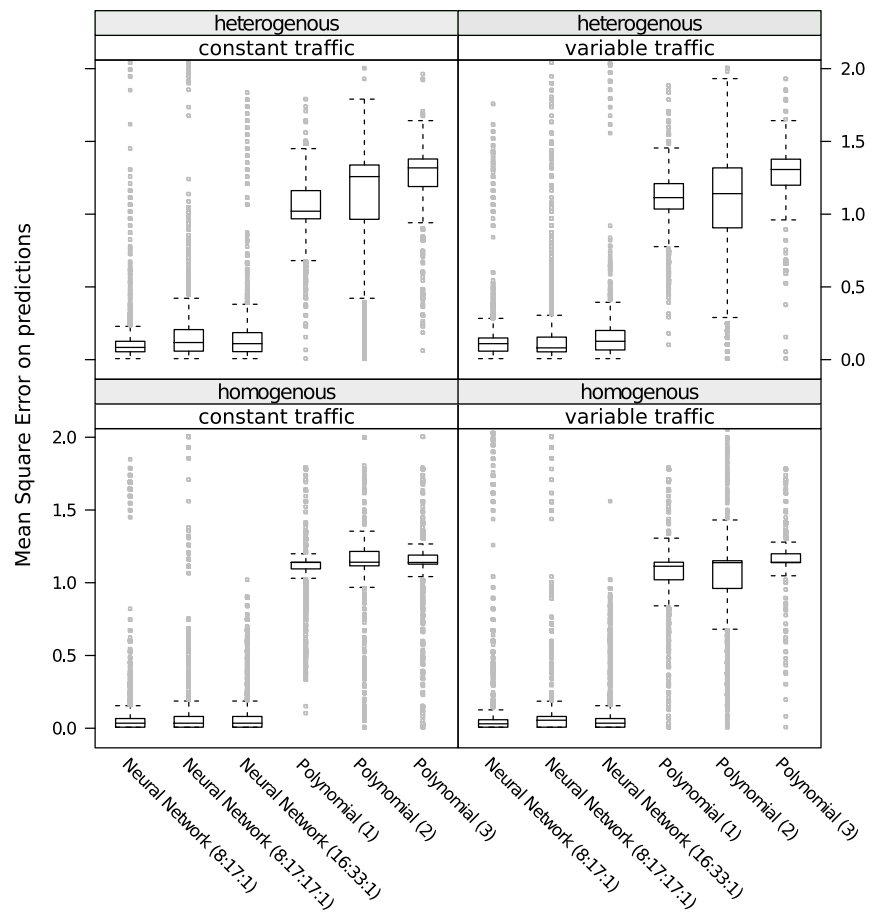

Fig. 3: Mean Square Error shown as histograms for the different descriptive models and under different circumstances in the four scenarios outlined in Section IV

Analyzing the results shown in Figure 3 shows two trends besides the apparent difference in performance between both types of models. A first trends is that the variability of the mean square error in the heterogenous scenarios is larger than that in the homogenous scenarios, as is illustrated by the height of the boxes in the boxplots. A second trend, especially among the polynomial models, is that the variability in the mean square error is larger for the variable traffic flow.

Based on these preliminary observations, it is clear that the neural network based models outperform the polynomial models. The remainder of the result analysis focusses on the performance of each of the neural network based models.

Figure 4 shows pairs of graphs for two of the scenarios. The first of each pair shows the evolution of the mean normalized square error as a function of the simulation time. These graphs show that as the model obtains more training data, the error on the predictions decreases. The second of each pair shows the evolution of the mean normalized square error in function of the prediction horizon, i.e. how far the prediction is in the future. These graphs show that as the prediction horizon increases, the predictions become more unreliable.

The scattering of grey data points is a good indication for the unreliability of the predictions as the horizon increases. Even as the trend line in some of the prediction horizon graphs is downward, the spread of actual averages greatly increases, thus indicating that the predictions became more unreliable.

\section{CONCLUSION AND FUTURE WORK}

This paper evaluates two models to generate ad hoc link traversal time predictions in a distributed multi-agent system, namely a polynomial model and an artificial neural network model. Microscopic traffic simulations where used to compare the predictions generated by both models with the actual observed link traversal times. The evaluation suggests that the artificial neural network model succeeds in learning the relationship between the number of entering vehicles and their link traversal times better than the polynomial model does.

Using the artificial neural network, road agents are able to use observations to build the model needed to generate ad hoc link traversal times. By aggregating the information contributed by vehicle agents and using that as the input for the learned model, road agents can generate the predictions and send them back to the vehicle agents. Vehicle agents can use these predictions to better inform the road agents of their estimated arrival times.

The evaluation provided in this paper can be considered the first step in finding good road models for ad hoc link traversal time prediction. The experiments show that artificial neural networks are capable of providing adequate predictions. Still, a more in depth evaluation of the use of artificial neural networks is required to find optimal neural network layouts, training techniques and inputs as well as the conditions, such as penetration rates for both infrastructure agents and vehicle agents, required for this approach to function.

As the number of different approaches discussed in the related work section illustrate, numerous methods of gen- 

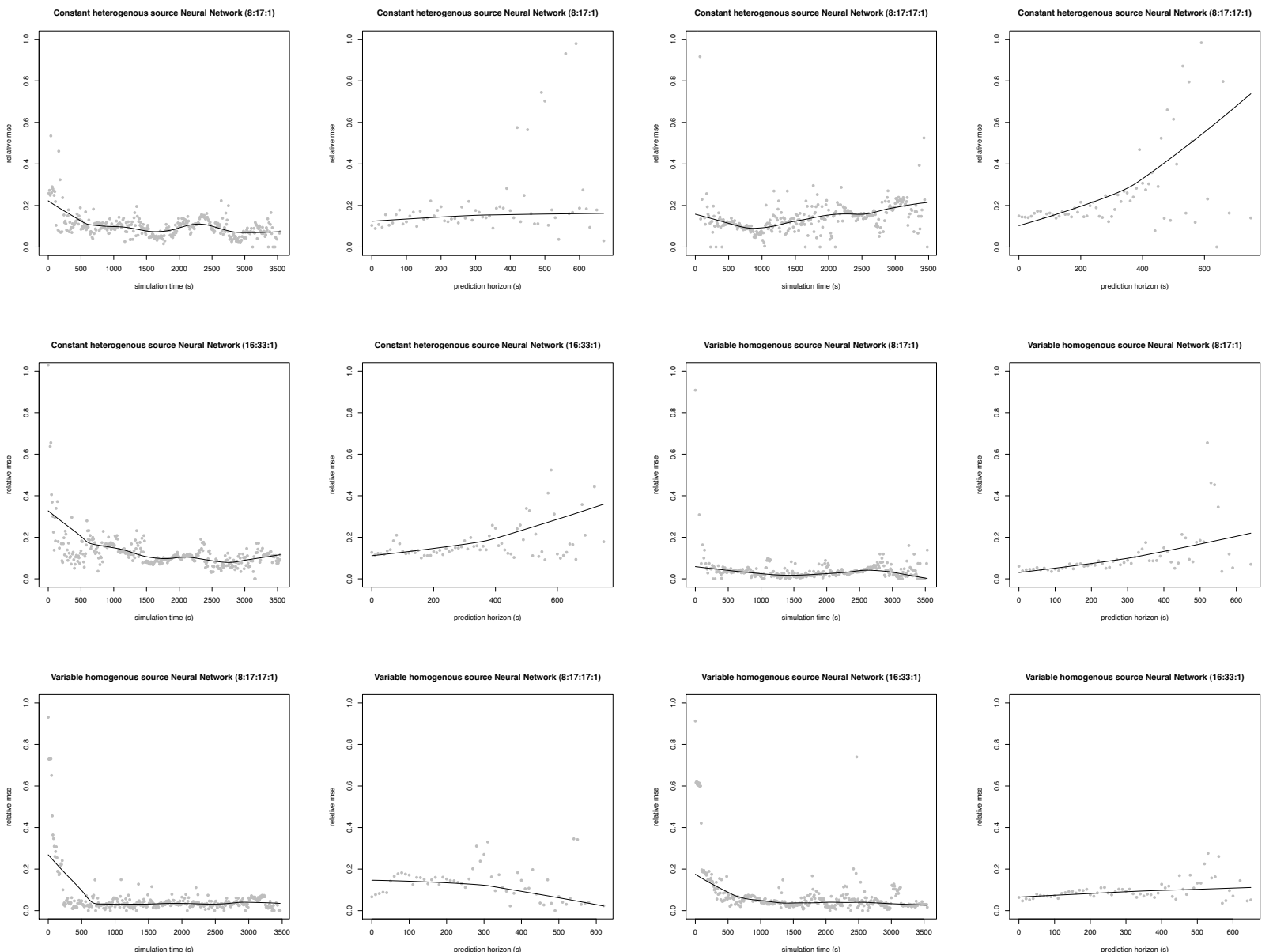

Fig. 4: Mean square error in function of both the simulation time and the prediction horizon. Grey dots represent average normalized mean square errors for predictions during a one second interval. The black smooth line shows the trend in the data.

erating link traversal times exist. One of the extension points for the approach described in this paper would be to include external information: information coming from neighboring road agents or other entities. Such a cooperation between road agents could further improve the reliability of the predictions by accounting for spill overs and other phenomena. The artificial neural network approach should be flexible enough to accommodate these additional inputs.

\section{REFERENCES}

[1] K. Wunderlich, D. Kaufman, and R. Smith, "Link travel time prediction for decentralized route guidance architectures," IEEE Transactions on Intelligent Transportation Systems, vol. 1, no. 1, pp. 4-14, 2000.

[2] J. Wahle, A. L. C. Bazzan, F. Klügl, and M. Schreckenberg, "Decision dynamics in a traffic scenario," Physica A: Statistical Mechanics and its Applications, vol. 287, no. 3-4, pp. 669 - 681, 2000.

[3] R. Claes and T. Holvoet, "Maintaining a distributed symbiotic relationship using delegate multiagent system," in Proceedings of the 2010 Winter Simulation Conference, B. Johansson, S. Jain, J. MontoyaTorres, J. Hugan, and E. Yücesan, Eds., 2010.

[4] R. Yasdi, "Prediction of road traffic using a neural network approach," Neural Computing amp; Applications, vol. 8, pp. 135-142, 1999, 10.1007/s005210050015. [Online]. Available: http://dx.doi.org/10.1007/s005210050015
[5] R. Claes, T. Holvoet, and D. Weyns, "A decentralized approach for anticipatory vehicle routing using delegate multi-agent systems," 2011, accepted for publication in IEEE Transactions on Intelligent Transportation Systems.

[6] [Online]. Available: http://commons.apache.org/math/

[7] [Online]. Available: http://neuroph.sourceforge.net/

[8] W. Lee, S. Tseng, and W. Shieh, "Collaborative real-time traffic information generation and sharing framework for the intelligent transportation system," Information Sciences, vol. 180, no. 1, pp. 6270, 2010.

[9] E. Horvitz, J. Apacible, R. Sarin, and L. Liao, "Prediction, expectation, and surprise: Methods, designs, and study of a deployed traffic forecasting service," in Twenty-First Conference on Uncertainty in Artificial Intelligence. Citeseer, 2005.

[10] M. M. Haklay and P. Weber, "OpenStreetMap: User-Generated street maps," IEEE Pervasive Computing, vol. 7, no. 4, pp. 12-18, 2008.

[11] R. Claes and T. Holvoet, "Gridlock: a microscopic traffic simulation platform," in 2nd International Conference on Models and Technologies for ITS, 2011.

[12] M. Treiber, A. Hennecke, and D. Helbing, "Congested traffic states in empirical observations and microscopic simulations," Phys. Rev. E, vol. 62, no. 2, pp. 1805-1824, Aug 2000.

[13] A. Kesting, M. Treiber, and D. Helbing, "General lane-changing model mobil for car-following models," Transportation Research Record: Journal of the Transportation Research Board, vol. 1999, no. -1, pp. 86-94, 01 2007. [Online]. Available: http://dx.doi.org/10.3141/199910 